\title{
Seroepidemiology of Human Cystic Echinococcosis Among Nomads of Lorestan Province, Iran
}

\author{
Ali Chegeni Sharafi, ${ }^{1}$ Farnaz Kheirandish, ${ }^{2,}{ }^{*}$ Mehrdad Valipour, ${ }^{3}$ Mohammad Saki, ${ }^{1}$ Elham Nasiri, ${ }^{4}$ and \\ Sara Darjazini ${ }^{1}$ \\ ${ }^{1}$ Health Department, Razi Herbal Medicines Research Center, Lorestan University of Medical Sciences, Khorramabad, Iran \\ ${ }^{2}$ Department of Medical Parasitology and Mycology, Razi Herbal Medicines Research Center, Lorestan University of Medical Sciences, Khorramabad, Iran \\ ${ }^{3}$ Department of Epidemiology and Statistics, Iran University of Medical Sciences, Tehran, Iran \\ ${ }^{4}$ Shahid Madani Hospital, Lorestan University of Medical Sciences, Khorramabad, Iran \\ "Corresponding author: Department of Medical Parasitology and Mycology, Razi Herbal Medicines Research Center, Lorestan University of Medical Sciences, Khorramabad, \\ Iran. E-mail: kheirandish81@yahoo.com
}

Received 2016 September 28; Revised 2017 October 17; Accepted 2017 October 22.

\begin{abstract}
Background: Cystic Echinococcosis (CE) is one of the major zoonotic parasitic diseases caused by the larval stages of parasite Echinococcus granulosus. This is an endemic disease in a number of regions in Iran, such as Lorestan province, and is considered as a health problem.

Objectives: Due to the close contact of nomadic people with sheep dog, this research aimed at studying the seroepidemiology of CE among in-migration and out-migration nomads of Lorestan province, Iran.

Methods: In this cross-sectional study, among 5920 residents of nomadic areas, 315 people were randomly selected and serum samples were obtained from these individuals. IgG antibodies against CE were evaluated using the Enzyme Linked Immunosorbent Assay (ELISA). Demographic data of all subjects were collected by a questionnaire, and the results were statistically analyzed using SPSS V.20 and STATA12.

Results: According to the results, the seroepidemiology of CE among the nomads was 18 (5.7\%); 13 (72.22\%) were male and five (27.77\%) were female yet the difference was not statistically significant. The highest prevalence (7.79\%) was in the age group of 20 to 30 years old, yet no significant difference was observed between various age groups. In terms of education, more seropositive cases were found amongst subjects with low education, yet no significant difference was observed between educational level and seropositivity of CE. It should also be noted that all the participants were not aware of proper washing of vegetables and fruits.

Conclusions: This study was conducted for the first time in Lorestan province, Iran, and showed that the seroprevalence among the nomadic population of Aligoudarz and Sepiddasht was considerable. In order to prevent zoonotic disease, increased awareness of nomads in understanding the transmission ways of disease is suggested through health education programs according to the educational level of the target population, the majority of whom are illiterate. Active surveillance is also recommended in these areas.
\end{abstract}

Keywords: Cystic Echinococcosis, Lorestan, Nomad, Seroprevalence

\section{Background}

Cystic Echinococcosis (CE) is one of the transmissible major diseases between animals and humans (1). The disease is common in most parts of the world, especially in countries with flourished animal husbandry. This disease causes much health and economic losses, annually (14 ). The disease causative agent is Echinococcus granulosus parasite, regarded as the second important parasitic worm disease in the world with global emission (5). Cystic Echinococcosis larval infection is caused by the growth of metacestode cysts in the intermediate host. The grown metacestodes in the organs of intermediate host typically generate a lot of protoscolices, and each of them has the potential to become adult worms in the body of the final host, since the infected organs are eaten (6-9). Studies have reported an average prevalence of $24.41 \%, 8.51 \%, 18.89 \%$, and $35.76 \%$ in sheep, goat, bison, and buffalo, respectively (10). The infection of dog, as the definitive host of the disease, has been reported from $5 \%$ to $49 \%$ in various parts of Iran, and also $27.8 \%$ in sheep dogs (1). The disease has been reported from most provinces of Iran. In Iran, average surgical cases is estimated 1.2 per 100000 people (8). The overall direct and indirect annual costs of diagnosis, treatment, 
and control of CE are estimated as US\$ 232.25 million in Iran, which indicates the importance of the issue (11). On the other hand, $60 \%$ of the infected rural population remains asymptomatic $(10,11)$.

Due to the spread of infection in various parts of the body and lack of a certain diagnosis method, serologic methods are quite useful in the diagnosis of the disease (2). The most appropriate immunoglobulin for detection of the history of CE or exposure to parasite is IgG, because the level remains high for a long time in blood $(9,12-16)$.

Enzyme Linked Immunosorbent Assay (ELISA), one of the serological methods for detection of hydatidosis with advantages, such as high sensitivity and specificity and implementation for many samples at the same time, is an appropriate method for seroepidemiological studies. Many researchers have reported on the prevalence of human hydatidosis by ELISA from several provinces of Iran (17-20).

\section{Objectives}

Considering the close contact of nomadic people with sheep dog, the present study was conducted to investigate the seroepidemiology of CE among in-migration and outmigration nomads in Lorestan province.

\section{Methods}

\subsection{Study Area}

Lorestan province is located in Western Iran, with an area of $28294 \mathrm{~km}^{2}$ and population of 1754000 . Lorestan province is the thirteenth province in Iran in terms of population, and is considered as one of the most populous provinces of Iran. The city of Khorramabad is the capital of the province. Lorestan province is a mountainous land and apart from a few deserts, it is totally covered by the Zagros Mountains. Lorestan province is also the third province in terms of water with $12 \%$ of the water in Iran. This province, because of vast mountains, meadows, and proper precipitation, is the habitat for people, whose main occupation is animal husbandry and agriculture.

\subsection{Sample Collection}

In this cross-sectional study, among 5920 people living in the in-migration and out-migration nomadic areas in Aligudarz and Sepiddasht in Lorestan province, a total of 315 people from August to November in 2016 were randomly selected and demographic data, such as age, gender, occupation, education, drinking water, washing method of vegetables, and history of contact with dogs were completed using the questionnaire. Then, in order to investigate the presence of IgG antibodies, $5 \mathrm{~mL}$ of blood sample was provided and located inside the cold box, and sent as soon as possible under cold chain conditions to the nearest health center laboratory for centrifugation and serum production. The serum samples were stored at $-20{ }^{\circ} \mathrm{C}$ until use.

\subsection{Enzyme Linked Immunosorbent Assay Test}

The collected sera were examined in terms of IgG antibodies against CE, using the Echinococcus ELISA Kit (Pishtazteb), in accordance with the manufacturer's protocol. All the samples at the same time and same condition were evaluated by ELISA. The absorbance was measured by an ELISA reader at $450 \mathrm{~nm}$ and reference wave of $630 \mathrm{~nm}$. The samples with optical density $10 \%$ higher than the specified cut-off were considered positive in terms of specific IgG against CE. The data were statistically analyzed using SPSS version 20 and STATA 12. T-test and chi-square were also used.

\section{Results}

Among 315 subjects, 161 (51.1\%) were male and 154 (48.9\%) were female. The most frequent age group was 10 to 20 years old, which constituted almost $25 \%$ of the participants. The seroprevalence of CE among the nomads was $5.7 \%$. Table 1 shows the description of characteristics of the participants in this study. Among 18 (5.7\%) subjects, who were serologically positive, 13 (72.22\%) were male and five $(27.77 \%)$ were female. Although the chance of infection with CE in males was 2.61 times more than females, yet the difference was not statistically significant $(\mathrm{P}<0.065)$. In this study, $82.22 \%$ of infections were found in the age group under 30 years, and the highest prevalence percentage $(7.79 \%)$ was in the age group of 20 to 30 , yet no significant difference was seen between the age groups $(\mathrm{P}<$ 0.719).

In terms of education, more seropositive cases were seen amongst subjects with low education, yet no significant difference was observed between educational level and seropositivity of $\mathrm{CE}(\mathrm{P}<0.546)$. All the subjects with a positive serum had direct contact with dogs, failure to properly wash fruits and vegetables, had no knowledge of the disease and also all of them used spring water for drinking and washing vegetables. In terms of occupation, all cases were working in agriculture and animal husbandry (Table 1).

\section{Discussion}

The hydatid cyst is one of the major zoonotic diseases frequently seen in most tropical and semi-tropical areas 


\begin{tabular}{|c|c|c|c|}
\hline Variable & No. of Samples & $\begin{array}{c}\text { Frequency of } \\
\text { Contamination (\%) }\end{array}$ & P Value \\
\hline \multicolumn{4}{|l|}{ Gender } \\
\hline Male & 161 & $13(8.07 \%)$ & 0.09 \\
\hline Female & 154 & $5(3.26 \%)$ & \\
\hline \multicolumn{4}{|l|}{ Level of education } \\
\hline Illiterate & 194 & $9(4.64 \%)$ & 0.55 \\
\hline Low literate & 106 & $8(7.55 \%)$ & \\
\hline Diploma & 15 & $1(6.67 \%)$ & \\
\hline \multicolumn{4}{|l|}{ Age group (years) } \\
\hline$<10$ & 75 & $5(6.67 \%)$ & 0.72 \\
\hline $10-19$ & 81 & $5(6.17 \%)$ & \\
\hline $20-29$ & 77 & $6(7.79 \%)$ & \\
\hline $30-39$ & 47 & $1(2.13 \%)$ & \\
\hline $40-49$ & 16 & $0(0 \%)$ & \\
\hline$\geq 50$ & 19 & $1(5.26 \%)$ & \\
\hline
\end{tabular}

in the world, especially in areas where a close relationship was seen between humans and dogs. Annually, many people are at risk of serious complications and even death caused by the disease $(5,6,21-23)$. In order to assess the prevalence of the disease in Iran, several studies have been based on hospital information or seroepidemiology data $(10,24)$. Rafie et al. showed the prevalence of human hydatid cysts in Khuzestan province was $13.8 \%$ by ELISA (7). Sarkari et al. reported that the prevalence of human hydatidosis in Yasujas was 7.2\% by ELISA (25). In the serological study of human CE in Kerman, the prevalence was 8.8\% (12). Mirnejade-Asl and Fasihi Harandi, in a seroepidemiological study of CE using ELISA in Mugan plain, Iran, reported the prevalence of hydatidosis infection as 9.2\% (26). The prevalence of CE in dogs as the certain host was $2 \%$ to $26 \%$, and in intermediate host (sheep, goats, calves, and camels), it was also reported as $1.5 \%$ to $70 \%(8,17,27,28)$.

This seroepidemiological study was also conducted to complete the available information about CE for the first time in the nomads of Lorestan province with limited access to medical facilities. According to the results, the percentage of positive CE serum cases was estimated $6.77 \%$. Serum positivity in males (55.56\%) was higher than females (44.44\%), yet no significant difference was seen between positive sero-epidemiology and gender $(\mathrm{P}<0.065)$.

Zibaie et al. (2011) reported the seroprevalence of 15.4\% in Khorramabad city, Lorestan province; the results are not in agreement with the current study. One of the reasons might be that people in Khorramabad city use vegetables from farms around the city, where the stray dogs are often found, while the nomadic people usually use mountain vegetables, and the access of stray dogs to these vegetables is lower (27). However, on the other hand, Zibaie et al. showed that most positive cases were aged 20 to 29 years old, which is consistent with the present study (27).

Due to difficult access to nomadic people that sometimes might require long walking distance with ice supply limitation to keep the cold chain of the samples, and restriction of vehicles to travel to these areas, this study is the first study in Lorestan province and might be one of the few studies in Iran that examined CE seroprevalence in the nomadic population. Determining the prevalence of $\mathrm{CE}$ and publishing these results could be effective to sensitize relevant authorities in various areas of medical and veterinary sciences to provide the required facilities and equipment for disease control and prevention. Thus, it seems that complementary studies in livestock and humans are required. In general, according to the results, in order to prevent and control the zoonotic disease, increased awareness of nomads in understanding the transmission ways of the disease is suggested through health education programs corresponding the educational level of the target population, the majority of whom are illiterate. Active surveillance is also recommended in these areas.

\section{Acknowledgments}

The authors would like to appreciate the Health Deputy of Lorestan University of Medical Sciences and Centre for Communicable Diseases Management for funding the project, as well as the personnel of Department of Communicable Diseases of Aligoudarz and Sepiddasht.

\section{References}

1. Eslami A, Hosseini SH. Echinococcus granulosus infection of farm dogs of Iran. Parasitol Res. 1998;84(3):205-7. [PubMed: 9521009].

2. Schantz PM. Parasitic zoonoses in perspective. Int J Parasitol. 1991;21(2):161-70. doi:10.1016/0020-7519(91)90006-s.

3. Eckert J, Gemmell MA, Meslin FX, Pawlowski ZS; World Health Organization. WHO/OIE manual on echinococcosis in humans and animals : a public health problem of global concern. Paris, France: World Organisation for Animal Health; 2001.

4. Budke CM, Deplazes P, Torgerson PR. Global socioeconomic impact of cystic echinococcosis. Emerg Infect Dis. 2006;12(2):296-303. doi: 10.3201/eid1202.050499. [PubMed: 16494758]. [PubMed Central: PMC3373106].

5. Torgerson PR, Shaikenov BS, Rysmukhambetova AT, Ussenbayev AE, Abdybekova AM, Burtisurnov KK. Modelling the transmission dynamics of Echinococcus granulosus in dogs in rural Kazakhstan. Parasitology. 2003;126(Pt 5):417-24. [PubMed: 12793645]. 
6. Eckert J, Deplazes P. Biological, epidemiological, and clinical aspects of echinococcosis, a zoonosis of increasing concern. Clin Microbiol Rev. 2004;17(1):107-35. [PubMed: 14726458]. [PubMed Central: PMC321468].

7. Mirzanejad-Asl H, fasihi harandi M. [Seroepidemiological Survey of Human Cystic Echinococcosis with ELISA Method in Moghan Plain, Ardabil Province].J Ardabil Univ Med Sci. 2009;9(4):334-46. Persian.

8. Dalimi A, Motamedi G, Hosseini M, Mohammadian B, Malaki H, Ghamari Z, et al. Echinococcosis/hydatidosis in western Iran. Vet Parasitol. 2002;105(2):161-71. doi: 10.1016/s0304-4017(02)00005-5.

9. Shariatzadeh SA, Spotin A, Gholami S, Fallah E, Hazratian T, MahamiOskouei M, et al. The first morphometric and phylogenetic perspective on molecular epidemiology of Echinococcus granulosus sensu lato in stray dogs in a hyperendemic Middle East focus, northwestern Iran. Parasit Vectors. 2015;8:409. doi: 10.1186/s13071-015-1025-9. [PubMed: 26242729]. [PubMed Central: PMC4526285].

10. Rokni MB. Echinococcosis/hydatidosis in Iran. Iran J Parasitol. 2009;4(2):1-16.

11. Fasihi Harandi M, Budke CM, Rostami S. The monetary burden of cystic echinococcosis in Iran. PLoS Negl Trop Dis. 2012;6(11). e1915. doi: 10.1371/journal.pntd.0001915. [PubMed: 23209857]. [PubMed Central: PMC3510083].

12. Rafiei A, Hemadi A, Maraghi S, Kaikhaei B, Craig PS. Human cystic echinococcosis in nomads of south-west Islamic Republic of Iran. 2007. Contract No.: 1020-3397.

13. Babba H, Messedi A, Masmoudi S, Zribi M, Grillot R, Ambriose-Thomas $P$, et al. Diagnosis of human hydatidosis: comparison between imagery and six serologic techniques. Am JTrop Med Hyg. 1994;50(1):64-8. [PubMed: 8304574].

14. Wen H, Craig PS. Immunoglobulin G subclass responses in human cystic and alveolar echinococcosis. Am JTrop Med Hyg.1994;51(6):741-8. [PubMed: 7810806].

15. Jiang L, Wen H, Ito A. Immunodiagnostic differentiation of alveolar and cystic echinococcosis using ELISA test with 18-kDa antigen extracted from Echinococcus protoscoleces. Trans Royal Soc Trop Med Hyg. 2001;95(3):285-8. doi: 10.1016/s0035-9203(01)90235-4.

16. Shambesh MK, Craig PS, Wen H, Rogan MT, Paolillo E. IgG1 and IgG4 serum antibody responses in asymptomatic and clinically expressed cystic echinococcosis patients. Acta Tropica. 1997;64(1-2):53-63. doi: 10.1016/s0001-706x(96)00637-7.

17. Heidari Z, Mohebali M, Zarei Z, Aryayipour M, Eshraghian M, Kia E, et al. Seroepidemiological study of human hydatidosis in meshkinshahr district, ardabil province, iran. Iran J Parasitol. 2011;6(3):19-25. [PubMed: 22347293]. [PubMed Central: PMC3279891].
18. Rakhshanpour A, Harandi MF, Moazezi S, Rahimi M, Mohebali M Mowlavi G, et al. Seroprevalence of human hydatidosis using ELISA method in qom province, central iran. Iran J Parasitol. 2012;7(3):10-5 [PubMed: 23109956]. [PubMed Central: PMC3469166].

19. Craig PS, Rogan MT, Allan JC. Detection, Screening and Community Epidemiology of Taeniid Cestode Zoonoses: Cystic Echinococcosis, Alveolar Echinococcosis and Neurocysticercosis. Adv Parasitol. 1996;38:169-250. doi: 10.1016/s0065-308x(08)60035-4.

20. Auer H, Picher O, Aspock H. Combined Application of Enzymelinked Immunosorbent Assay (ELISA) and Indirect Haemagglutination Test (IHA) as a Useful Tool for the Diagnosis and Post-operative Surveillance of Human Alveolar and Cystic Echinococcosis. Zentralblatt fur Bakteriologie, Mikrobiologie und Hygiene. Series A: Med Microbiol Infect Dis Virol Parasitol. 1988;270(1-2):313-25. doi: 10.1016/s01766724(88)80169-x.

21. McManus DP, Zhang W, Li J, Bartley PB. Echinococcosis. Lancet. 2003;362(9392):1295-304. doi: 10.1016/s0140-6736(03)14573-4.

22. Moro P, Schantz PM. Echinococcosis: a review. Int J Infect Dis 2009;13(2):125-33. doi:10.1016/j.ijid.2008.03.037. [PubMed: 18938096]

23. Craig PS, McManus DP, Lightowlers MW, Chabalgoity JA, Garcia $\mathrm{HH}$, Gavidia CM, et al. Prevention and control of cystic echinococcosis. Lancet Infect Dis. 2007;7(6):385-94. doi: 10.1016/s1473-3099(07)70134-2.

24. Rouhani S, Parvizi P, Spotin A. Using specific synthetic peptide (p176) derived AgB 8/1-kDa accompanied by modified patient's sera: a novel hypothesis to follow-up of Cystic echinococcosis after surgery. Med Hypotheses. 2013;81(4):557-60. doi: 10.1016/j.mehy.2013.07.003. [PubMed: 23890801].

25. Sarkari B, Sadjjadi SM, Beheshtian MM, Aghaee M, Sedaghat F. Human cystic echinococcosis in Yasuj District in Southwest of Iran: an epidemiological study of seroprevalence and surgical cases over a ten-year period. Zoonoses Public Health. 2010;57(2):146-50. doi: 10.1111/j.1863-2378.2008.01200.x. [PubMed: 19175567].

26. Asghari M, Mohebali M, Kia EB, Farahnak A, Aryaeipour M, Asadian S, et al. Seroepidemiology of Human Hydatidosis Using AgB-ELISA Test in Arak, Central Iran. Iran J Public Health. 2013;42(4):391-6. [PubMed: 23785678]. [PubMed Central: PMC3684725].

27. Zibaei M, Azargoon A, Ataie-Khorasgani M, Ghanadi K, Sadjjadi SM The serological study of cystic echinococcosis and assessment of surgical cases during 5 years (2007-2011) in Khorram Abad, Iran. Niger J Clin Pract. 2013;16(2):221-5. doi: 10.4103/1119-3077.110156. [PubMed: 23563466].

28. Yousefi Darani H, Avijgan M, Karimi K, Manouchehri K, Masood J. Seroepidemiology of hydatid cyst in Chaharmahal va Bakhtiari Province, Iran. Iran J publ health. 2003;32(2):31-3. 